\title{
Lower- versus upper-extremity surgery: effect on serum albumin levels
}

İrfan AYDIN ${ }^{1}$, İsmail AĞIR²

\footnotetext{
${ }^{1}$ Department of Emergency Medicine, Faculty of Medicine, Adiyaman University Adiyaman, Turkey

${ }^{2}$ Department of Orthopedics and Traumatology, Faculty of Medicine, Adiyaman University, Adiyaman, Turkey.
}

\section{ABSTRACT}

Hypoalbuminemia is one of the markers of trauma and major surgery. No study has reported on the comparison between upper- and lower-extremity surgery patients in terms of albumin levels. Therefore, the present study aimed to investigate the preoperative and postoperative serum albumin levels in patients who underwent extremity surgery and compare the changes in albumin levels after upper-extremity surgery with those after lower-extremity surgery.

Sixty patients who presented to the emergency department of a tertiary care hospital due to trauma were included in the study. All the patients underwent either upper- or lower-extremity surgery by orthopedists and were consecutively selected regardless of their ages. Their serum albumin levels at the time of admission and on the third day after the surgery were obtained from the hospital patient records.

A statistically significant difference in the preoperative and postoperative serum albumin levels was found in both groups $(P<0.05)$. Moreover, the mean difference between preoperative and postoperative serum albumin levels was found to be significantly higher in the lower-extremity group $(1.52 \pm 0.32 \mathrm{~g} / \mathrm{dL})$ than in the upper-extremity group $(0.95 \pm 0.39 \mathrm{~g} / \mathrm{dL})$ $(P<0.05)$.

This study revealed that both upper- and lower-extremity surgeries caused hypoalbuminemia. This study was novel in finding that lower-extremity surgery caused more severe hypoalbuminemia in the patients compared with upper-extremity surgery. The reason for this significant difference was the longer duration of lower-extremity surgeries, requiring more fluid support during the surgery.

Key words: Falls from a height; hypoalbuminemia; lower-extremity surgery; serum albumin level; trauma surgery; upperextremity surgery

\section{INTRODUCTION}

Major surgical interventions and traumas cause severe stress responses in human metabolism. This metabolic stress leads to higher rates of surgical complications and delays in recovery. In many cases, metabolic stress causes morbidity and mortality (1). Some inflammatory markers, such as C-reactive protein and albumin, help determine the metabolic condition and predict the prognosis of patients (1-4).

Albumin, a plasma protein, is synthesized in the liver and responsible for some main functions, including maintaining colloid osmotic pressure that keeps the body fluid in the intravascular space, showing antithrombotic effect, binding and transporting some agents, scavenging the free oxygen radicals, and playing a role on vascular permeability in shock and sepsis $(5,6)$.

The serum albumin level decreases in two ways: (1) by decrease in the synthesis of albumin in the liver by chronic liver diseases, malnutrition, malabsorption, and carcinomas; and (2) by increased loss of albumin through traumas, nephrotic 
syndrome, major bleeding, fistulas, and glomerulonephritis, and in pregnancy and drug use $(7,8)$.

Studies have reported that hypoalbuminemia is one of the markers for trauma and major surgery $(3,4,9,10)$. A few studies have focused on extremity surgery due to trauma and hypoal buminemia. No studies have reported on the comparison between upper- and lower-extremity surgery patients in terms of hypoalbuminemia.

Therefore, the present study aimed to investigate the preoperative and postoperative serum albumin levels in patients who underwent extremity surgery and compare the changes in albumin levels after upper-extremity surgery with those after lower-extremity surgery for the first time in the literature.

\section{METHODS}

The present study was approved by the ethics committee (October 23, 2018; reference number 2018/7-4).

\section{Patients}

A total of 60 patients, who were referred to the emergency department of a tertiary care hospital, with trauma such as traffic accidents, falls, and falls from a height between March 31, 2016, and March 31, 2018, were enrolled into the study retrospectively. All the patients included in the study underwent upper- or lower-extremity surgery by the department of orthopedics and traumatology, and were consecutively selected regardless of their ages. In addition, patients with only acute traumatic surgery and one extremity surgery were selected. Those who had multiple trauma or underwent multiple surgeries were excluded from the study. Patients who underwent both upper- and lower-extremity surgeries were also excluded. Routine orthopedic surgery patients were excluded as well.

The patients were divided into 2 groups: upper-extremity surgery (30 patients) and lower-extremity surgery (30 patients).

\section{Tests}

The serum albumin levels at the time of admission and on the third day after the surgery were obtained from the hospital patient records. They were analyzed using a particle-enhanced turbidimetric inhibition immunoassay method with an Architect c8000 auto-analyzer (Abbott Laboratories, IL, USA). The serum albumin level of the patients was tested each day during hospitalization. All the patients in this study stayed at the hospital for at least 3 days, and most of them (32/60) stayed only for 3 days. Therefore, the albumin levels of the days 0 and 3 were noted for a standardized interpretation.

Statistical analysis

Continuous variables were expressed as mean \pm standard deviation. The normality test of the numerical variables was checked using the Kolmogorov-Smirnov test. The independent-samples $t$ test was used to compare two independent groups. The paired-samples t test was used to compare pre- and postoperative albumin values. Data were statistically analyzed using the software Jamovi project (2018; Jamovi version 0.9.2.6; retrieved from https://www.jamovi. org, open source). A $P$ value less than 0.05 was considered as statistically significant.

\section{RESULTS}

The upper-extremity surgery group consisted of 17 (56.7\%) male and 13 (43.3\%) female patients, and the lower-extremity surgery group had 16 (53.3\%) male and 14 (46.7\%) female patients. No statistically significant differences between the preoperative and postoperative serum albumin levels were found $(1.31 \pm 0.43 \mathrm{~g} / \mathrm{dL}$ in male and $1.14 \pm 0.48 \mathrm{~g} / \mathrm{dL}$ in female; $P=0.150$ ).

The mean age of the patients who underwent upper- and lowerextremity surgeries was $43.20 \pm 22.05$ years (between 11 and 80 years) and $42.76 \pm 20.97$ years (between 6 and 76 years), respectively. No statistically significant correlation between ages of the patients and differences in preoperative and postoperative serum albumin levels was found $(r=-0.239$ and $P=0.202$ in the lower-extremity surgery group; $r=0.070$ and $P=0.714$ in the upper-extremity surgery group).

The mean hospitalization period was $3.93 \pm 1.43$ days (min: 3 , max: 9) for the patients in the upper-extremity surgery group and 


\begin{tabular}{lll} 
TABLE 1: Distribution of the patients according to the reasons of the fractures. \\
\cline { 2 - 3 } & \multicolumn{2}{c}{ Group (Number of patients) } \\
\cline { 2 - 3 } & Lower extremity & Upper extremity \\
\hline Falls & 14 & 14 \\
Out-car traffic accidents & 7 & 4 \\
Falls from height & 2 & 6 \\
In-car traffic accidents & 2 & - \\
Motorcycle accident & 2 & 1 \\
Other & 3 & \\
\hline
\end{tabular}

\section{$4.4 \pm 1.86$ days (min: 3 , max: 10) in the lower-extremity surgery} group. No significant difference in terms of hospital stay was observed between the two groups $(P>0.05)$.

The most common reason for the fractures was falls in both groups (14 patients for both groups). The other common reasons were falls from height and out-car and in-car traffic accidents (Table 1).

Three patients in the upper-extremity surgery group had open humerus shaft fractures: two of these had Gustili Anderson type 2 and one had type 3a. Among the 27 patients with closed fractures, 11 patients had humerus shaft fractures, 6 had humerus supracondylar fractures, 4 had humerus proximal fractures, 2 had humerus medial epicondyle fractures, and 4 had clavicula, ulna, or radius fractures.

In the lower-extremity surgery group, two patients had open femur shaft fractures. Among the 27 patients with closed fractures, 7 had multiple femur and/or tibia fractures, 5 had tibia shaft fractures, 3 had femur shaft fractures, 2 had tibia-fibula fractures, 2 had tibial plateau fractures, 7 had single tibial fractures in various segments, and 2 had single femur fractures.

The serum albumin levels were normal before the surgery but decreased after the surgery in both groups. A statistically significant difference in terms of preoperative and postoperative serum albumin levels was noted in both groups $(P<0.05)$ (Table 2$)$.

The mean difference between preoperative and postoperative serum albumin levels was found to be $0.95 \pm 0.39 \mathrm{~g} / \mathrm{dL}$ in the upper-extremity surgery group and $1.52 \pm 0.32 \mathrm{~g} / \mathrm{dL}$ in the lowerextremity surgery group. The mean difference between these two groups was statistically significant $(P<0.05)$, indicating that the lower-extremity surgery patients had a significantly higher decrease in albumin levels (Table 2).

TABLE 2: Comparison between the groups in terms of preoperative and postoperative albumin levels.

\begin{tabular}{|c|c|c|c|}
\hline & \multicolumn{2}{|c|}{ Group } & \multirow[t]{2}{*}{$P$} \\
\hline & Lower extremity & Upper extremity & \\
\hline Preoperative albumine level (g/dL) & $3.76 \pm 0.37$ & $3.65 \pm 0.38$ & $0.293 a$ \\
\hline Postoperative albumin level (g/dL) & $2.24 \pm 0.28$ & $2.71 \pm 0.47$ & $<0.05 a$ \\
\hline $\mathrm{P}^{\mathrm{b}}$ & $<0.05 b$ & $<0.05 b$ & \\
\hline Difference in albumin levels & $1.52 \pm 0.32$ & $0.95 \pm 0.39$ & $<0.05 a$ \\
\hline
\end{tabular}




\section{DISCUSSION}

Hypoalbuminemia is a well-known marker of malnutrition. It has also a prognostic and predictive value for many diseases or conditions such as major surgery, carcinomas, trauma, and colorectal or gastrointestinal disorders. Decreased serum albumin levels show a direct influence on the increase in the length of hospital stay, complications of surgery, morbidity, and even mortality (5,11-16). Hypoalbuminemia is reported to be associated with poor tissue healing and collagen synthesis, as well as malnutrition. In addition, it is a good marker of traumatic, cardiac, colorectal, and general surgery $(5,16)$.

The present study showed that upper- and lower-extremity surgeries were associated with postoperative hypoalbuminemia. In addition, the study showed that the serum albumin levels were significantly decreased in the surgery of lower extremity compared with the upper extremity.

Several studies reported an association between malnutrition and major surgery. Studies showed that hypoalbuminemia was a marker of malnutrition in the patients who underwent major surgery. However, in the present study, all the patients of both groups had the same nutrition program. In addition, some studies showed that plasma albumin levels might decrease in spite of aggressive replacement therapy in some major trauma patients. They explained that the condition was due to the altered endothelial permeability caused by cytokines such as interleukin-1 (16-19). Thus, this study showed that the hypoalbuminemia occurred due to not only malnutrition but also some other factors.

Stress response after surgery and trauma has been a well-known entity, and it involved emerging metabolic, hormonal, and electrolytic changes, and release of cytokines. It was stated that albumin showed an immediate response to surgical stress (1, 9, 20). Hübner et al. (1) found that postoperative decrease in serum albumin reflected the magnitude of surgery and the associated stress response. In major trauma and surgeries, excessive fluid leaks into the interstitial and intravascular spaces as a result of systemic and tissue reactions, thereby decreasing the serum albumin levels $(3,21)$. Protein metabolism is reported to be significantly disturbed after any kind of traumatic event such as surgery, sepsis, and burn injuries. Albumin has been identified as a reliable marker of this process. Serum concentrations of albumin showed a significant decrease as early as a few hours after the trauma or surgery $(1,4,10)$. In the present study, it was accepted that all the patients had trauma and stress and all underwent a major surgery. This explained the cause of hypoalbuminemia in all patients who participated in this study; however, the significantly higher hypoalbuminemia in the lower-extremity surgery patients was not clearly understood as it was not known which of the two surgeries caused more stress.

One of the major differences between the patient groups in this study was immobility. Patients who underwent lower-extremity surgery had much more immobility duration than the ones who had upper-extremity surgery. Despite the fact that Afshinnia et al. (22) stated that immobility and hypoalbuminemia are closely associated with osteoporosis, no evidence about an association between hypoalbuminemia and immobility of the patients was found. Immobility in the lower-extremity surgery patients was considered to cause more severe hypoalbuminemia in this study, although enough evidence was lacking.

In the present study, three patients had open fractures in the upper-extremity surgery group and two in the lower-extremity surgery group. However, multiple close fractions were more in the lower-extremity surgery group. This could be a difference between the groups causing a higher albumin decrease due to an effect on increasing metabolism as a result of trauma and injury (2).

The other major difference between the patient groups was the duration of the surgery. The lower-extremity surgery took significantly more time compared with the upper-extremity surgery. Therefore, more fluid support was given to the patients in the lower-extremity surgery groups during the surgery. More liquid support results in the dilution of plasma, and hence a decreased serum albumin level. In this context, the given excessive fluid was rapidly eliminated, and the overload of sodium was slowly ejected, resulting in further elongated extra fluid dilution 
of albumin. Studies have shown that the severity of the catabolic damage caused a proportional increase in vascular permeability (23). Therefore, in the present study, the duration of surgery of the lower extremities and the higher amount of fluid given during the surgery might explain the decrease in albumin.

Older patients who underwent surgery had more severe hypoalbuminemia compared with the younger patients (3). However, in the present study, no association was found between the age of the patients and serum albumin levels. In addition, no relationship was found between the serum albumin levels and the gender of patients.

This study had some limitations. The patients were followed up only for the first 3 days, not until the discharge. In addition, the serum albumin level was observed only as a laboratory finding, the clinical reflection of hypoalbuminemia was ignored.

\section{CONCLUSIONS}

In conclusion, the upper- and lower-extremity surgeries were found to cause hypoalbuminemia. In addition, this study was novel in finding that lower-extremity surgery caused more severe hypoalbuminemia in the patients compared with the upperextremity surgery regardless of the association with some factors such as age, gender, nutrition, and stress. The causative factor for this significant difference could be explained as the long duration of lower-extremity surgeries, requiring more fluid support during the surgery.

\section{REFERENCES}

1. Hübner M, Mantziari S, Demartines N, Pralong F, Coti-Bertrand P, Schäfer M. Postoperative Albumin Drop Is a Marker for Surgical Stress and a Predictor for Clinical Outcome: A Pilot Study. Gastroenterol Res Pract 2016;2016:8743187.

2. Marik $P E, M$. Flemmer $M$. The immune response to surgery and trauma: implications for treatment. J Trauma Acute Care Surg 2012;73:801-8

3. Alberti LR, Petroianu A, Veloso DFM. Theeffect of surgicaltrauma in post-operative albumin concentration after major surgeries. Einstein (Sao Paulo) 2016;7:48-51.

4. Rittler $P$, Jacobs $R$, Demmelmair $H$, Kuppinger D, Braun $S$, Koletzko $B$, Jauch KW, Hartl WH. Dynamics of albumin synthesis after major rectal operation. Surgery 2007;141:660-6.
5. Truong A, Hanna MH, Moghadamyeghaneh Z, Stamos MJ. Implications of preoperative hypoalbuminemia in colorectal surgery. World J Gastrointest Surg 2016;8:353-362.

6. Don BR, Kaysen G. Serum albumin: relationship to inflammation and nutrition. Semin Dial 2004;17:432-7.

7. Kim S, McClave SA, Martindale RG, Miller KR, Hurt RT. Hypoalbuminemia and Clinical Outcomes: What is the Mechanism behind the Relationship? Am Surg 2017;83:1220-7.

8. Sindgikar V, NarasanagiB,Tejasvini V, Ragate A, Patel FA. Effect of serum albumin in wound healing and its related complications in surgical patients. Al Ameen J Med Sci 2017;10:132-5.

9. Russell JA. Management of sepsis. N Engl J Med 2006;355:1699713.

10. Moghadamyeghaneh $\mathrm{Z}$, Hanna $\mathrm{MH}$, Carmichael JC, Nguyen NT, Stamos MJ. A nationwide analysis of postoperative deep vein thrombosis and pulmonary embolism in colon and rectal surgery. J Gastrointest Surg 2014;18:2169-77.

11. Lee JI, Kwon M, Roh JL, Choi JW, Choi SH, Nam SY, Kim SY. Postoperative hypoalbuminemia as a risk factor for surgical site infection after oral cancer surgery. Oral Dis 2015;21:178-84.

12. Fujiwara $Y$, Shiba $H$, Shirai $Y$, Iwase $R$, Haruki $K$, Furukawa K, Futagawa $Y$, Misawa T, Yanaga K. Perioperative serum albumin correlates with postoperative pancreatic fistula after pancreaticoduodenectomy. Anticancer Res 2015;35:499-503.

13. Labgaa I, Joliat GR, Demartines N, Hübner M. Serum albumin is an early predictor of complications after liver surgery. Dig Liver Dis 2016;48:559-61.

14. Sang BH, Bang JY, Song JG, Hwang GS. Hypoalbuminemia within two postoperative days is an independent risk factor for acute kidney injury following living donor liver transplantation: a propensity score analysis of 998 consecutive patients. Crit Care Med 2015:43:2552-61.

15. Lee EH, Chin JH, Choi DK, Hwang BY, Choo SJ, Song JG, Kim TY, Choi IC. Postoperative hypoalbuminemia is associated with outcome in patients undergoing off-pump coronary artery bypass graft surgery. J Cardiothorac Vasc Anesth 2011;25:4628.

16. Labgaa I, Joliat G-R, Kefleyesus A, Mantziari S, Schäfer $M$, Demartines $N$, Hübner $M$. Is postoperative decrease of serum albumin an early predictor of complications after major abdominal surgery? A prospective cohort study in a European centre. BMJ Open 2017;7:e013966.

17. Sun J, Sun F, Wang $X$, Yuan $S$, Zheng $S$, Mu X. Risk factors and prognosis of hypoalbuminemia in surgical septic patients. Peer J 2015;3:e1267.

18. Gatta A, Verardo A, Bolognesi M. Hypoalbuminemia. Intern Emerg Med. 2012;7:S193-9.

19. Ryan S, Politzer C, Fletcher A, Bolognesi M, Seyler $T$. Preoperative hypoalbuminemia predicts poor short-term outcomes for hip fracture surgery. Orthopedics. 2018; https:// doi.org/10.3928/01477447-20180912-03.

20. Ryan AM, Hearty A, Prichard RS, Cunningham A, Rowley SP, Reynolds JV. Association of hypoalbuminemia on the first postoperative day and complications following esophagectomy. J Gastrointest Surg 2007;11:1355-60. 
21. Iannuzzi JC, Chandra A, Rickles AS, Kumar NG, Kelly KN, Gillespie DL, Monson JR, Fleming FJ. Resident involvement is associated with worse outcomes after major lower extremity amputation. J Vasc Surg 2013;58:827-31.e1.

22. Afshinnia F, Wong KK, Sundaram B, Ackermann RJ, Pennathur
S. Hypoalbuminemia and Osteoporosis: Reappraisal of a Controversy. J Clin Endocrinol Metab 2016;101:167-75.

23. Redelmeier DA. New thinking about postoperative hypoalbuminemia: a hypothesis of occult protein-losing enteropathy. Open Med 2009;3:e215-9. 